\title{
Double minimum in the surface stabilized ferroelectric liquid crystal switching response
}

\author{
C. V. Brown ${ }^{\text {a) }}$ and J. M. Hind \\ School of Science, The Nottingham Trent University, Clifton Lane, \\ Nottingham NG11 8NS, United Kingdom \\ K. P. Lymer \\ QinetiQ Farnborough, Cody Technology Park, Ively Road, Farnborough, \\ Hampshire GUI4 OLX, United Kingdom \\ J. C. Jones \\ ZBD Displays Limited, The Malvern Hills Science Park, Geraldine Road, Great Malvern, \\ Worcestershire WR14 3SZ, United Kingdom
}

(Received 25 March 2004; accepted 28 June 2004)

\begin{abstract}
A double minimum has recently been observed in the time-voltage switching response for a smectic $\mathrm{C}^{*}$ liquid crystal layer in the surface stabilized geometry ("Ferroelectric Liquid Crystal Device," K. P. Lymer and J. C. Jones, U.K. Patent No. GB2338797, 17th June 1999). Liquid crystal continuum theory is used to demonstrate that this unusual switching behavior arises if the equilibrium orientation of the molecular director rotates around the smectic cone as a function of distance through one half of the layer only. The double minimum is shown to evolve for large differences between the $\varepsilon_{2}$ and $\varepsilon_{1}$ components of the smectic C biaxial permittivity tensor. (C) 2004 American Institute of Physics. [DOI: 10.1063/1.1786365]
\end{abstract}

The fast, bistable, electro-optical switching response of surface stabilized ferroelectric liquid crystal (SSFLC) devices ${ }^{1}$ has been exploited for applications including miniature displays for camera viewfinders ${ }^{2}$ and in high resolution, polarization insensitive, diffractive optical elements. ${ }^{3,4}$ The SSFLC exploits the strong coupling between an applied electric field $E$ and the spontaneous polarization $P_{S}$ of the smectic $\mathrm{C}^{*}$ material. The chiral pitch of the material is unwound, provided the layer thickness is much less than the pitch, due to the strong alignment interaction with the unidirectionally rubbed polymer surfaces that coat the confining plates.

An internal layer structure that can result from this type of confinement is depicted in Fig. 1. The thick lines in the side view (a) show the molecular director, i.e., the local optical axis, which lies on the smectic cone of half-angle $\theta$. The smectic layers form a chevroned structure with layer tilt angles of $\pm \delta$. The equilibrium orientation of the director can vary through the thickness of the cell in several ways, and some possible director profiles are depicted by the positions of the filled circles in Figs. 1(b)-1(e). The director orientation at a given position $z$ in the layer is described by the azimuthal angle $\phi(z)$. The direction of the $P_{S}$ vector along the $\mathrm{C}_{2}$ symmetry axis and at the tangent to the smectic cone is illustrated at one director position.

Smectic C materials are inherently biaxial, with large differences measured between the three principle permittivities: $\varepsilon_{3}$ along the liquid crystal molecular director which is typically the long axis, $\varepsilon_{2}$ parallel to the $\mathrm{C}_{2}$ symmetry axis, and $\varepsilon_{1}$ which is mutually perpendicular to $\varepsilon_{2}$ and $\varepsilon_{3}{ }^{5-7}$ In materials where the dielectric biaxiality $\partial \varepsilon=\varepsilon_{2}-\varepsilon_{1}$ is high, typically $\partial \varepsilon>0.5$, the electro-optical switching response of an SSFLC layer can show a minimum. ${ }^{8}$ This occurs due to

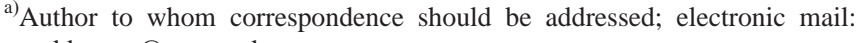
carl.brown@ntu.ac.uk
}

the competition between the coupling of the applied electric field to the spontaneous polarization $P_{S}$ and to the biaxial dielectric permittivity. The $P_{S}$ coupling promotes switching, and this dominates at low field, whereas the dielectric coupling usually impedes switching, and this dominates at high voltages.

Figure 2 shows time-voltage switching curves from Ref. 9 for a high biaxiality smectic $\mathrm{C}^{*}$ material in the SSFLC geometry. Curve (a) shows a single minimum and this was observed for Nylon 6,6 surface alignment layers. In curve (b), however, there is a double minimum where the voltage minima are separated by a sharp peak. This unusual switching curve resulted from using thin polyimide (probimide 32) alignment layers. The dramatic differences between the two types of switching behavior that are observed for the same smectic $\mathrm{C}^{*}$ material can be explained in terms of differences

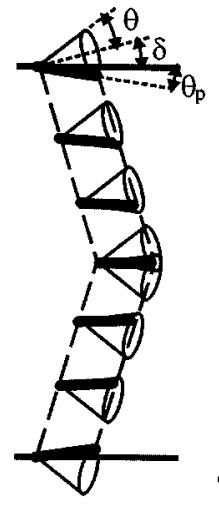

(a)

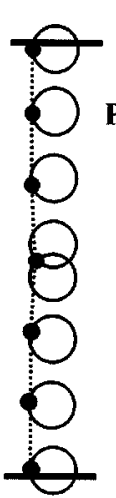

(b)

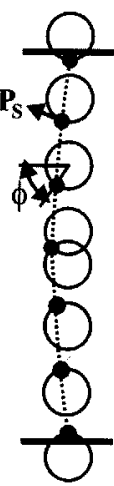

(c)

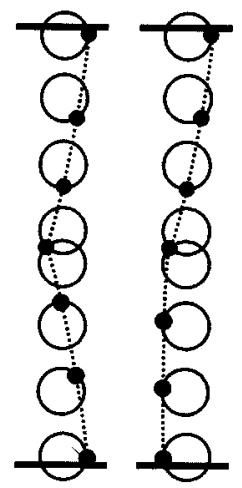

(d) (e)
FIG. 1. Illustration of the surface stabilized ferroelectric liquid crystal layer geometry. The chevron structure, layer tilt angle $\delta$, and smectic cone angle $\theta$ are depicted in the side view (a). End views are shown for the cases where the azimuthal angle at the surfaces, $\phi_{\mathrm{SU}} / \phi_{\mathrm{SL}}$, were as follows: (b) $0^{\circ} / 0^{\circ}$, (c) $90^{\circ} /-90^{\circ}$, (d) $180^{\circ} /-180^{\circ}$, and (e) $180^{\circ} / 0^{\circ}$. 


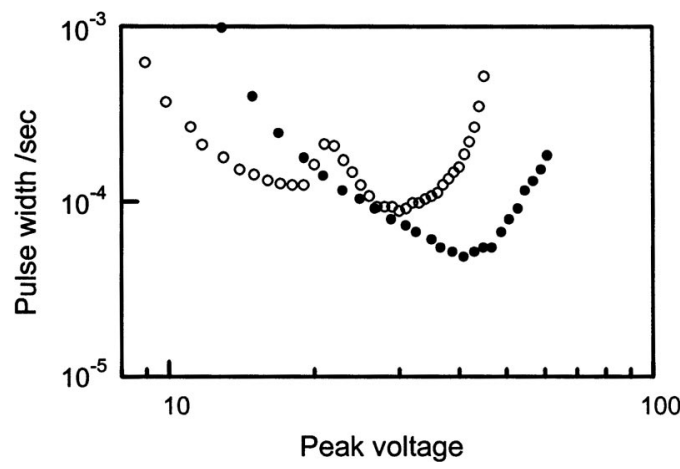

FIG. 2. Time-voltage switching curve (taken from Ref. 9). Curve (a), closed circles, shows a single minimum and corresponds to a rubbed nylon alignment layer. Curve (b), open circles, shows two minima with a sharp cusp between them. This was observed for the same material but with a polyimide alignment layer.

in the through-layer equilibrium director profiles caused by the different alignment layers.

Several possible director profiles at equilibrium, i.e., with no applied voltage, are illustrated in Figs. 1(b)-1(e). In each case, the lower of the two possible bistable of the director at the chevron cusp, $\phi(z=d / 2)=(\pi / 2) \pm \phi_{\text {ch }}$, is shown where $\phi_{\text {ch }} \arcsin (\tan \delta / \tan \theta)$. The differences between the director profiles arise due to the differences in the positions of the director at the upper surface, $\phi_{\mathrm{SU}}$, and at the lower surface, $\phi_{\mathrm{SL}}$. Figures 1(b)-1(d) show the cases of $\phi_{\mathrm{SU}} / \phi_{\mathrm{SL}}$ $=0^{\circ} / 0^{\circ} /, 90^{\circ} /-90^{\circ}$, and $180^{\circ} / 180^{\circ}$, respectively. For typical values of $\delta$ and $\theta$ (Ref. 7), the director profile is the most uniform in Fig. 1(b) and the least uniform, with a large in-plane twist from the surface to the chevron cusp, in Fig. $1(\mathrm{~d})$. Figure 1(e) shows the case where $\phi_{\mathrm{SU}} / \phi_{\mathrm{SL}}=180^{\circ} / 0^{\circ}$. This is distinct in that the director profile is uniform in the upper half of the layer, but twisted, or "half-splayed" in the lower half. In a real device, the different profiles in Fig. 1 can arise depending on whether the layer tilt $\delta$ has the same sign as the tilt of the director at the surface, $\theta_{p}$, as with the $\mathrm{C} 1$ geometries in Figs. 1(b), 1(d), and 1(e), or it has the opposite sign, as with the $\mathrm{C} 2$ geometry in Fig. 1(c). ${ }^{8}$

A theoretical model based on liquid crystal continuum theory has been used to generate time-voltage switching curves for pulsed voltages applied across the SSFLC layer in the geometries shown in Fig. 1. The time evolution of the liquid crystal director, $\phi(z, t)$, has been calculated by solving the discretized form of Eq. (1) on a linear array of points using a numerical relaxation technique. ${ }^{7-10}$

$$
\begin{aligned}
\eta \frac{\partial \phi}{\partial t}= & B\left(\frac{\partial^{2} \phi}{\partial z^{2}}\right)+P_{S} E \cos \delta \sin \phi \\
& -\frac{1}{2} \varepsilon_{0} E^{2} \partial \varepsilon \sin 2 \phi \cos ^{2} \delta-\frac{1}{2} \varepsilon_{0} E^{2}\left(\varepsilon_{3}\right. \\
& \left.-\varepsilon_{1}\right) \cos \phi\left(\frac{1}{4} \sin 2 \theta \sin 2 \delta\right. \\
& \left.-\sin \phi \cos ^{2} \delta \sin ^{2} \phi\right)
\end{aligned}
$$

Where $B$ is an isotropic elastic constant and $\eta$ is the viscosity for rotation around the smectic cone. The electric field profile, $E(z)$, was obtained at each time step by integration of the Maxwell equation, $\nabla \cdot \boldsymbol{D}=0$. With variations in the $z$ direction

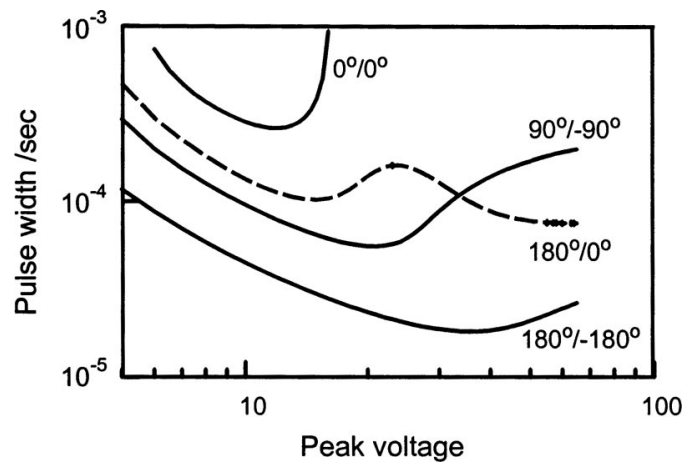

FIG. 3. Time-volatge switching curves generated by the continuum simulation with the parameters from Table I for the geometries shown in Fig. 1. Values for $\phi_{\mathrm{SU}} / \phi_{\mathrm{SL}}$ are shown next to the appropriate curve.

only the displacement field is given by $D_{z}=\varepsilon_{0} \varepsilon_{z z}(z) E(z)$ $+P_{z}(z)$ where $\varepsilon_{z z}(z)$ and $P_{z}(z)$ are the $z$ components of the dielectric permittivity tensor and the polarization vector, respectively.

The director was fixed at the surfaces, $\phi(z=0)=\phi_{\mathrm{SU}}$ and $\phi(z=d)=\phi_{\mathrm{SL}}$. The azimuthal angle $\phi$ of the director at $z$ $=d / 2$ was calculated by assuming that the free energy at the chevron interface, $W_{\mathrm{ch}}$, is given by Eq. (2) Similar phenomenological expressions have been used in literature to model the SSFLC time response and to reproduce single minimum SSFLC time-voltage curves ${ }^{11,12}$

$$
W_{\mathrm{ch}}=W_{0}\left(\left[\frac{\phi-\pi / 2}{\phi_{\mathrm{ch}}}\right]^{2}-1\right)^{2}
$$

Figure 3 shows time-voltage switching curves that have been generated by the numerical solution of Eqs. (1) and (2) as function of time using the parameters shown in Table I. The equilibrium profiles in Figs. 1(b)-1(e) were each used as the starting point for the simulation at time $t \leqslant 0$. A voltage was applied at time $t=0$ with a polarity that acts to increase the azimuthal angle $\phi$ by coupling to the spontaneous polarization. A significant increase in $\phi$ across the bulk region between the chevron and the surface-in either half of the layer-causes a switching torque to be exerted at the chevron interface. A torque of sufficient magnitude causes the director to switch across to the higher of the two possible bistable position, $\phi(z=d / 2)=(\pi / 2)+\phi_{\mathrm{ch}}$, at the chevron cusp. The pulse width is defined here as the time taken for the director at the chevron interface to the switch to the halfway point, $\phi(z=d / 2)=\pi / 2$, at each voltage.

TABLE I. Values used for the dielectric, elastic, and physical parameters of the smectic $\mathrm{C}^{*}$ liquid crystal in the numerical simulations.

\begin{tabular}{ccc}
\hline \hline Symbol & Value & Name \\
\hline$\varepsilon_{1}$ & 5.0 & Permittivity \\
$\partial \varepsilon$ & 1.15 & Dielectric biaxiality \\
$\Delta \varepsilon$ & -2.0 & Dielectric anisotropy \\
$P_{S}$ & $6.6 \times 10^{-5} \mathrm{C} \mathrm{m}^{-2}$ & Spontaneous polarization \\
$\delta$ & $21.0^{\circ}$ & Layer tilt angle \\
$\theta$ & $25.0^{\circ}$ & Smectic C cone angle \\
$B$ & $1.0 \times 10^{-11} \mathrm{~N}$ & Elastic constant \\
$W_{O}$ & $2.0 \times 10^{-4} \mathrm{~N} \mathrm{~m}^{-1}$ & Chevron energy \\
$d$ & $1.5 \times 10^{-6} \mathrm{~m}$ & Layer thickness \\
\hline \hline
\end{tabular}




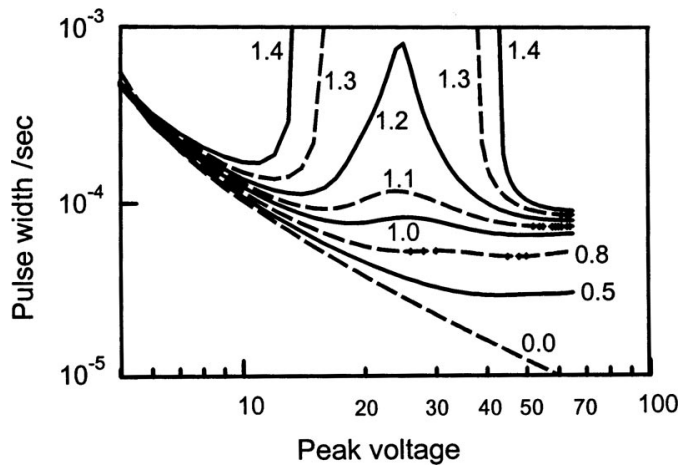

FIG. 4. Time-voltage switching curves generated by the continuum simulation for the geometries shown in Fig. 1 with $\phi_{\mathrm{SU}} / \phi_{\mathrm{SL}}=180^{\circ} / 0^{\circ}$. The parameters from Table I were used except for the dielectric biaxiality $\delta \varepsilon$ which was varied between $\delta_{\varepsilon}=0.0$ (lower curve) and $\delta_{\varepsilon}=1.4$ (upper curve) as indicated.

The curves for which the initial director profile is symmetrical about the chevron interface, i.e. when $\phi_{\mathrm{SU}} / \phi_{\mathrm{SL}}$ $=0^{\circ} / 0^{\circ}, 90^{\circ} /-90^{\circ}$, and $180^{\circ} /-180^{\circ}$, all show a similar shape with a single minimum. However, the position of that minimum moves to a higher voltage and a lower pulse width time as $\left|\phi_{\mathrm{SU}}\right|$ (which equals $\left|\phi_{\mathrm{SL}}\right|$ ) is increased. Although the time evolution of the director profile within the layer in each case is complicated, this dependence can be explained by the nature of the coupling between the applied field and the dielectric permittivity. This coupling acts to maximize the component of the permittivity that is aligned with the field. The two values of $\phi$ for which this maximization would occur are $\phi=(\pi / 2) \pm \phi_{\mathrm{AC}}$, where $\phi_{\mathrm{AC}}$ is readily derived by allowing the value of $E$ to tend to infinity in Eq. (1). If the initial director angle in the bulk of the layer lies in the range $\phi<\pi / 2$, then the dielectric coupling acts to stabilize the director angle toward $\phi=(\pi / 2)-\phi_{\mathrm{AC}}$, whereas if bulk director angle is predominantly in the range $\phi>\pi / 2$, then this stabilization is toward the higher angle, $\phi=(\pi / 2)+\phi_{\mathrm{AC}}$. The former case obtains for the $\left|\phi_{\mathrm{SU}}\right|=0^{\circ}$ profile in Fig. 1(b) for which the dielectric coupling impedes switching and increases the switching time. The opposite effect occurs in the latter case, exemplified by $\left|\phi_{\mathrm{SU}}\right|=180^{\circ}$, where switching to higher values of $\phi$ is favored, which reduces the switching time.

The curve for $\phi_{\mathrm{SU}} / \phi_{\mathrm{SL}}=180^{\circ} / 0^{\circ}$ Fig. 3 shows a double minimum. The equilibrium initial director profile from which this was calculated is depicted in Fig. 1(e). The lower half of the profile is the same as the nearly uniform $0^{\circ} / 0^{\circ}$ case in Fig. 1(b), and the upper half of the profile is the same as the twisted $180^{\circ} /-180^{\circ}$ profile in Fig. 1(d). The position of the maximum of the $180^{\circ} / 0^{\circ}$ switching curve lies at a time and voltage that is between the positions of the minima for the $0^{\circ} / 0^{\circ}$ and $180^{\circ} /-180^{\circ}$ curves. When a voltage is applied both sides of the layer exert a torque at the chevron interface. At lower voltages, there are contributions from the fast director reorientation from the upper half of the layer and the slower director reorientation in the lower half of the layer. At higher voltages, the lower half of the layer does not switch at all and the $180^{\circ} / 0^{\circ}$ curve tends towards the $180^{\circ} /-180^{\circ}$ curve. The extra maximum in the $180^{\circ} / 0^{\circ}$ curve arises at intermediate voltages when the switching torque from the lower half of the layer is tending to zero, but before the reorientation in the upper half dominates.

The evolution of the double minimum as a function of the dielectric biaxiality, $\partial \varepsilon$, is shown in Fig. 4 for the $180^{\circ} / 0^{\circ}$ curve. The dielectric biaxiality was varied between $\partial \varepsilon=0.0$ and $\partial \varepsilon=1.4$. At values up to $\partial \varepsilon=0.5$, only a single minimum is seen in the time-voltage switching curve. At values, above this, where $\partial \varepsilon>0.5$, the double minimum shape starts to evolve until a sharp peak is seen between the two minima at $\partial \varepsilon=1.2$. At still higher values, the switching curve is actually split into two regions and, remarkably, the pulse width time is infinite between the two regions. This strong dependence on the value of the biaxiality is consistent with the critical influence of the dielectric coupling to the applied field on the switching process.

${ }^{1}$ N. A. Clark and S. T. Lagerwall, Appl. Phys. Lett. 36, 899 (1980).

${ }^{2}$ N. A. Clark, C. Crandall, M. A. Handschy, M. R. Meadows, R. M. Malzbender, C. Park, and J. Z. Xue, Ferroelectrics 246, 1003 (2000).

${ }^{3}$ S. Warr and R. Mears, Electron. Lett. 31, 714 (1995).

${ }^{4}$ C. V. Brown and E. E. Kriezis, Appl. Opt. 42, 2257 (2003).

${ }^{5}$ S. J. Elston, J. R. Sambles, and M. G. Clark, J. Appl. Phys. 68, 1242 (1990).

${ }^{6}$ J. C. Jones, M. J. Towler, and E. P. Raynes, Ferroelectrics 121, 91 (1991).

${ }^{7}$ C. V. Brown and J. C. Jones, J. Appl. Phys. 86, 3333 (1999).

${ }^{8}$ J. C. Jones, M. J. Towler, and J. R. Hughes, Displays 14, 86 (1993).

${ }^{9}$ K. P. Lymer and J. C. Jones, UK Patent No. GB2338797 (17 June 1999). [Equivalent filings: U.S. Patent No. US6326940 (4 December 2001) and Japan Patent No. JP2000035563 (4 December 2001)].

${ }^{10}$ T. Carlsson, I. W. Stewart, and F. M. Leslie, J. Phys. A 25, 2371 (1992).

${ }^{11}$ J. E. Maclennan, M. A. Handschy, and N. A. Clark, Liq. Cryst. 7, 787 (1990).

${ }^{12}$ S. M. Said and S. J. Elston, Liq. Cryst. 28, 561 (2001). 
Applied Physics Letters is copyrighted by the American Institute of Physics (AIP). Redistribution of journal material is subject to the AIP online journal license and/or AIP copyright. For more information, see http:/ojps.aip.org/aplo/aplcr.jsp

Copyright of Applied Physics Letters is the property of American Institute of Physics and its content may not be copied or emailed to multiple sites or posted to a listserv without the copyright holder's express written permission. However, users may print, download, or email articles for individual use. 